\title{
Covariant Model for Relativistic Three-Body Systems *
}

\author{
Philippe Droz-Vincent \\ LUTH, Observatoire de Paris-Meudon \\ Place Jules Janssen, F-92195 Meudon, France
}

\begin{abstract}
The system is described by three mass-shell constraints. When at least two masses are equal, this picture has a reasonable nonrelativistic limit. At first post-Galilean order and provided the interaction is not too much energy-dependent, the relativistic correction is tractable like a conventional perturbation problem. A covariant version of harmonic oscillator is given as a toy model.
\end{abstract}

A system of three particles can be covariantly described by three massshell constraints, involving an interaction term referred to as potential. These constraints must reduce to three independent Klein-Gordon (or Dirac) equations in the absence of potential. In any case, they determine the evolution of a wave function which depends on three four-dimensional arguments, say $p_{a}$ with $a, b=1,2,3$, if we chose the momentum representation of quantum mechanics.

Naturally, the potential depends on both configuration and momentum variables, $q_{a}, p_{b}$, and must allow for mutual compatibility of the constraints. Moreover it happens that, just like in the Bethe-Salpeter approach, manifest covariance is paid by the presence of redundant degrees of freedom of

*Talk given at Quark Confinement and the Hadron Spectrum VI, Villasimius, Cagliari, Sardinia, Italy. 21-25 September 2004 
which the elimination is by no means straightforward (in contrast to the two-body case). These two important issues have been considered earlier by H. Sazdjian [1] who aimed at solving the general $n$-body case and proposed an approximate solution.

Specially dealing with the three-boson case, we have recently exhibited in closed analytic form a new set of variables $q_{a}^{\prime}, p_{b}^{\prime}$. In terms of these new variables, admissible expressions for the potential are explicitly available, and two superfluous degrees of freedom can be eliminated [2]. Setting $P=\sum p$ we linearly introduce relative variables

$$
z_{A}=q_{1}-q_{A}, \quad y_{A}=\frac{P}{3}-p_{A}, \quad A=2,3
$$

and similar formulas for $z_{A}^{\prime}, y_{B}^{\prime}$ in terms of $q_{a}^{\prime}, p_{b}^{\prime}$.

The mass-shell constraints can be equivalently replaced by their sum and differences; it is convenient to set $\nu_{A}=\frac{1}{2}\left(m_{1}^{2}-m_{A}^{2}\right)$.

The difference equations, in their original form, yield no simplification. But we perform a quadratic change among the momenta, say $p_{a} \rightarrow p_{a}^{\prime}$, or equivalently $P, y_{A} \rightarrow P^{\prime}, y_{A}^{\prime}$. in order to ensure the elimination of two redundant degrees of freedom; this change is characterized by

$$
\left(p_{1}-p_{A}\right)\left(p_{1}+p_{A}\right)=\left(p_{1}^{\prime}-p_{A}^{\prime}\right) \cdot P
$$

whereas $P^{\prime}=P$ and the transverse parts of the momenta remain unaffected, say $\widetilde{y}^{\prime}=\widetilde{y}$, where the tilda on any four-vector refers to its transverse part with respect to $P$.

Of course, this procedure generates a change of canonical variables [2], in particular we obtain new configuration variables, $z_{A}^{\prime}$.

\section{Three-dimensional Reduction}

We impose a sharp value of the total linear momentum, it is a timelike vector $k$, and we define $k \cdot k=M^{2}$.

Notations: The hat on any vector refers to its transverse part with respect to $k$.

Underlining any dynamical variable indicates that, in its expression, we substitute $k$ for $P$ and take into account equation the difference equations

$$
3 y_{A}^{\prime} \cdot k \Psi=\left(4 \nu_{A}-2 \nu_{B}\right) c^{2} \Psi
$$


We factorize out the relative energies; as a result the sum equation becomes

$$
\left(3 \sum m^{2}-M^{2}\right) c^{2} \psi=6\left(\widehat{y}_{2}^{2}+\widehat{y}_{3}^{2}+\widehat{y}_{2} \cdot \widehat{y}_{3}\right) \psi+\left(6 M^{2} c^{2} \underline{\Xi}+18 \underline{V}\right) \psi
$$

for a reduced wave function $\psi$ which depends only on the transverse relative momenta $\widehat{y}_{A}^{\prime}=\widehat{y}_{A}$.

The meaning of $\Xi$ is purely kinematic; this term depends only on the momenta and can be expressed in terms of their transverse part and $P$. Here $V$ denotes the relativistic potential; it may be phenomenological or motivated by considerations of field theory. In particular it may be formally constructed as a sum of two-body terms, like in equation (5) below; so doing one uses the shape of two-body potentials but (for the sake of compatibility) with the new three-body variables as arguments. Not only the total momentum $P$ but also the new configuration variables $z_{A}^{\prime}$ mix the two-body clusters, which amounts to automatically incorporate three-body forces. Admissible potentials entail that $\underline{V}$ is a function of the new variables $\widehat{z}_{2}^{\prime}, \widehat{z}_{3}^{\prime}$ and $M^{2} c^{2}$.

The reduced equation (2) is actually a nonconventional eigenvalue probem, where the operator to be diagonalized explicitly depends on its eigenvalue. This situation is by no means a special drawback of our model, in fact it is common in relativistic quantum mechanics [3], but it would make a general treatment rather involved.

On the other hand, it is natural to expand the formulas in powers of $1 / c^{2}$ and to look for the nonrelativistic limit. For arbitrary masses, the term $M^{2} c^{2} \underline{\Xi}$ generally blows up, which leads to consider, instead of (2) an alternative combination of the mass-shell constraints.

Two equal masses.

Drastic simplifications arise when two masses are equal, say $m_{2}=m_{3}=m$, equivalently $\nu_{2}=\nu_{3}=\nu$. We find that the Galilean limit of our eigenvalue problem is a Schroedinger equation with effective (or Galilean) masses that are generally distinct from the constituent masses $m_{a}$. However they still coincide with the constituent masses, at first order in the "mass-dispersion index" $\nu / m^{2}$.

Three Equal Masses.

When $m_{a}=m$ for all particles, equation (2) can be written as follows, using the rest frame

$$
\lambda \psi=\left(\mathbf{y}_{2}^{2}+\mathbf{y}_{3}^{2}+\mathbf{y}_{2} \cdot \mathbf{y}_{3}\right) \psi-3 \underline{V} \psi-M^{2} c^{2} \Xi \psi
$$


with $6 \lambda=\left(M^{2}-9 m^{2}\right) c^{2}$. Now the last term in (3) remains finite in the nonrelativistic limit. Indeed we can write $M c^{2} \Xi \underline{\Xi}=\frac{1}{M^{2} c^{2}} \Gamma_{(0)}+O\left(1 / c^{4}\right)$ where

$$
\Gamma_{(0)}=\frac{3}{4}\left\{\left(\widehat{y}_{2}^{2}\right)^{2}+\left(\widehat{y}_{3}^{2}\right)^{2}+4\left(\widehat{y}_{2} \cdot \widehat{y}_{3}\right)^{2}+2\left(\widehat{y}_{2}^{2}+\widehat{y}_{3}^{2}\right)\left(\widehat{y}_{2} \cdot \widehat{y}_{3}\right)-\widehat{y}_{2}^{2} \widehat{y}_{3}^{2}\right\}
$$

At first order in $1 / c^{2}$ we can, in $\Xi$, replace $M^{2}$ by $9 m^{2}$, which is independent from $\lambda$. Thus we replace $M^{2} c^{2} \Xi$ by $\Gamma_{(0)} / 9 m^{2} c^{2}$. If the relativistic "potential" $V$ doesnot depend on $P^{2}$, or if this dependence is of higher order, equation (3) becomes a conventional eigenvalue problem, tractable by perturbation theory. The last term in(3) brings a negative correction to the value $\lambda_{\mathrm{NR}}$ furnished by the nonrelativistic approximation, say

$$
\lambda=\lambda_{N R}-<\Gamma_{(0)}>/ 9 m^{2} c^{2}
$$

if $\lambda_{\mathrm{NR}}$ corresponds to a nondegenerate level. One has to calculate $\left\langle\Gamma_{(0)}\right\rangle$ in the eigenstate solution of the nonrelativistic problem.

\section{Harmonic Oscillator}

A covariant version of the harmonic potential is given by

$$
V=2 \kappa\left\{\left(\widetilde{z}_{2}^{\prime}\right)^{2}+\left(\widetilde{z}_{3}^{\prime}\right)^{2}-\widetilde{z}_{2}^{\prime} \cdot \widetilde{z}_{3}^{\prime}\right\}
$$

hence $\underline{V}$ in terms of $\widehat{z}_{A}^{\prime} \cdot \widehat{z}_{B}^{\prime}=-\mathbf{z}_{A}^{\prime 2} \cdot \mathbf{z}_{B}^{\prime 2}$. In the nonrelativistic limit we recover the naive $S U_{6}$ invariant Schroedinger equation. At the first postGalilean approximation, $M^{2}$ can be replaced by $9 \mathrm{~m}^{2}$, neglecting the dependence on total energy in the reduced equation. At this stage, the eigenvalue problem amounts to diagonalize a nonrelativistic harmonic oscillator, with potential $V_{\mathrm{NR}}=-3 \underline{V} / m$, submitted to a momentum-dependent perturbation. Expressed in terms of Jacobi-like coordinates, namely $\mathbf{R}_{2}=$ $-\mathbf{z}_{2}^{\prime}+\mathbf{z}_{3}^{\prime} \quad \mathbf{R}_{3}=\left(\mathbf{z}_{2}^{\prime}+\mathbf{z}_{3}^{\prime}\right) / \sqrt{3}$ and their conjugate momenta, the unperturbed ground state is a Gaussian. If the unit of lenght is choosen such that $\kappa=\frac{2}{9}$, one finds $<\Gamma_{(0)}>=11+1 / 4$.

This approach is intented for applications to confining interactions; future work should implement spin and investigate a possible contact with recents developments [4] of the BS approach. 


\section{References}

[1] H. Sazdjian, Physics Lett. B 208, 470(1988); Annals of Phys. 191, 52(1989).

[2] Ph. Droz-Vincent, Int. Jour. of Theor. Phys. 42, 1809 (2003).

[3] V.A. Rizov, H. Sazdjian, I.T.Todorov Ann. of Phys. 165, 59, (1985)

[4] J. Bijtebier, Jour. of Phys. G: Nucl. Part. Phys. 26, 871(2000) 